\title{
Measurement of Overall Performance Effectiveness in Setup Improvement
}

\section{Shye-Nee Low, Siu-Hou Chong, Hui-Yee Sim, Solehhudin Razalli, and Shahrul Kamaruddin}

School of Mechanical Engineering, Universiti Sains Malaysia, Engineering Campus, Nibong Tebal, 14300 Pulau Pinang, Malaysia

Correspondence should be addressed to Shahrul Kamaruddin; meshah@usm.my

Received 4 June 2014; Revised 20 August 2014; Accepted 26 August 2014; Published 11 September 2014

Academic Editor: Aydin Nassehi

Copyright (C) 2014 Shye-Nee Low et al. This is an open access article distributed under the Creative Commons Attribution License, which permits unrestricted use, distribution, and reproduction in any medium, provided the original work is properly cited.

\begin{abstract}
This study aimed to improve the setup process of injection molding machines by using the developed setup improvement methodology. Overall performance effectiveness (OPE) was used to evaluate the setup improvement. A case study was tested on the application of the developed setup improvement methodology. A 50.1\% reduction in setup time was attained by the developed methodology, and significant time savings were achieved with minimum investment. Comparisons between before and after improvement implementation were conducted through OPE to verify the improvement. In terms of OPE, the setup performance in the case study considered an acceptable value of $60.45 \%$. The setup process performance of the developed setup improvement methodology was judged in terms of effectiveness. Results therefore indicate that OPE measurement is an effective way to analyze the efficiency of a single setup process.
\end{abstract}

\section{Introduction}

Customer demands with low volume but high variety are often highly volatile because of the growing dynamics of today's market. Low-volume production for a high variety of goods reveals to companies the necessity of improving their changeover or setup performance to provide quick response to customer demands. Van Goubergen and Van Landeghem [1] argued that setup reduction is important as it can significantly affect production time to increase production throughput. Single-minute exchange of die (SMED) is a setup reduction concept introduced by Shigeo Shingo in the 1950s to improve productivity by improving setup operations. SMED is used as one of the elements in a continuous improvement process [2]. For instance, Patel et al. [3] developed a new structured methodology based on lean SMED and mistake proofing methods to improve the changeover process in the machining of precision components for the aerospace industry in a more efficient manner as well as to redesign these activities to make the convertibility process less timely by using simple poka-yoke devices. Cakmakci and Karasu [4] used a predetermined time system to standardize setup operations according to a set of standard operation procedures in the automobile industry. The integration of a predetermined time system and SMED yields a better improvement of setup operations based on a detailed motion study of setup activities. Moreira and Pais [5] showed that SMED is an excellent concept for improving setup operations in the mold-making industry. SMED implementation allowed the authors to identify the key drivers to productivity improvement and to improve setup operations by reducing waste activities, which represent about $2 \%$ of the industry's sales volume. These previous works proved that SMED is capable of improving setup activities in various industries.

Moxham and Greatbanks [6] claimed that the adoption of setup measurement in SMED enables companies to understand where they currently stand in setting up a process. The measurement indicator of setup performance is vital for measuring and monitoring the improvement of the setup process. Based on the wide applications of SMED, setup time reduction is the common measurement indicator of setup performance improvement [7]. Other measurement indicators used to measure setup improvement through SMED including process capability analysis [8], setup cost [9], and 
distance travelled by operators during the changeover process [10]. For example, Cakmakci [8] used the process capability analysis to quantify SMED capability in one process to indicate process variability and process deviation. However, none of the works on setup reduction focused on improving setup activities from the perspective of process effectiveness. The existence of effectiveness measurement techniques can differentiate the issues, problems, and potentials for improvement and development during the setup process in a short time frame. Therefore, a setup reduction approach that embraces performance measurement in terms of effectiveness must be developed to improve the setup process performance.

\section{Methodology}

Figure 1 illustrates the sequence of the setup reduction process. The process consists of two sections: setup improvement methodology and performance evaluation measurement. The first section assists the users in analyzing and improving the quick changeover operations to reduce setup time, while the second section focuses on measuring the effectiveness of the setup process before and after improvements.

Step 1 (study current setup). To initiate the setup realization process, understanding the production floor is a must. Most of the production floor will have a number of duplicate machines or groups of machines, so the setting up activities of every machine will be similar. Therefore, selecting the most important machine among the groups of machines is advisable, rather than focusing individually on each machine. The criteria for machine selection are based on its availability and usability, which can be identified through the historical data available on the shop floor. Once the machine is identified, details on the current setup activities need to be collected, such as a detailed motion and time study of the setup operations. The activities involved in setting up operations will be verified and recorded by a video recorder.

Step 2 (categorize setup). In Step 2, the activities of setup operations are separated according to the categories shown in Figure 2. The categories are used to identify the value-added (VA) activities, non-value-added but necessary (NVAN) activities, and non-value-added (NVA) activities responsible for performance in the setup operation. According to Ohno [11], VA activities are processes that add value to the product by altering the shape or character of a particular material, product, or assembly, such as by assembling parts into a functional part. NVAN activities are activities that are outside the standard of work, yet indirectly add value to the products, such as securing a mold in position with bolts and nuts during IM. NVA activities are regarded as waste in the conventional sense. Waste is considered an unproductive activity and is needless in any aspect. Waste should be removed once found, for example, traveling from the machine to the tool storage to pick up parts when a wrong part is brought along initially. The setup activities in the NVA category will be investigated in the next step because the improvement opportunity lies in the detailed operations of the setup.
Step 3 (evaluate the setup operation). Once the category of the setup operation is clearly identified, the information will be used to analyze the overall performance of the setup activities. The developed performance measurement, namely, OPE, originated from the concept of overall effectiveness equipment (OEE). OPE considers two measurement elements. The first element is the availability of a setup technician to set up the machine. Availability is the ratio of the total time of VA and NVAN activities to the total setup time (1). Total setup time is the actual setup time used in the entire setup process. Availability of a setup technician refers to the overall time spent on the machine setup with minimum wastage activities. VA activities can be evaluated by reducing the wastage activities. This step ensures that setup technicians will spend their time focusing on machine setup instead of on irrelevant setup procedures:

$$
\begin{aligned}
& \text { Availability } \\
& \qquad=\frac{[\text { Total Setup Time }(\text { mins })-\text { NVA Activities (mins) })}{[\text { Total Setup Time }(\text { mins })]} .
\end{aligned}
$$

The second element is the performance of setup operations by the technician. Performance is the ratio of discrepancy between the total setup times to the planned setup time (2). As planned setup time is the target time set by the management, the performance ratio can evaluate the capability of setup technicians to perform their work to achieve the set target. Performance ratio is used to evaluate the performance of technicians in achieving the planned time compared to their actual performance in setup operations. Thus, OPE is calculated by

$$
\begin{aligned}
& \text { Performance }=\frac{[\text { Planned Setup Time }(\text { mins })]}{[\text { Total Setup Time }(\text { mins })]}, \\
& \text { OPE }=(\text { Availability } * \text { Performance }) * 100 \% .
\end{aligned}
$$

Many researchers have suggested an ideal value for OEE; the average acceptance value proposed is between $60 \%$ and $70 \%[12,13]$. If the OPE value does not reach the acceptable range, then an improvement of the setup operation needs to be carried out, as elucidated in Step 4; otherwise, the deliberate setup activities are effective.

Step 4 (identify improvement opportunities). In Step 4, the improvement opportunity lies in the setup operation. It is carried out by investigating and analyzing the activities that have been categorized under NVA (Step 3). From the opportunity determination, the cause of the occurrence will be brainstormed, and alternatives for setup improvement will be generated. Reduction of setup time is achieved by improving the steps shown in Figure 3. In this study, NVAN activities are considered as VA activities that contribute indirectly to the setup operation. Without these activities, the setup operation will not be completed. For example, securing bolts on the mold is considered an NVA activity in the IM process; however, without the bolts, the mold will not attach firmly to the machine. Therefore, the elimination of NVA 


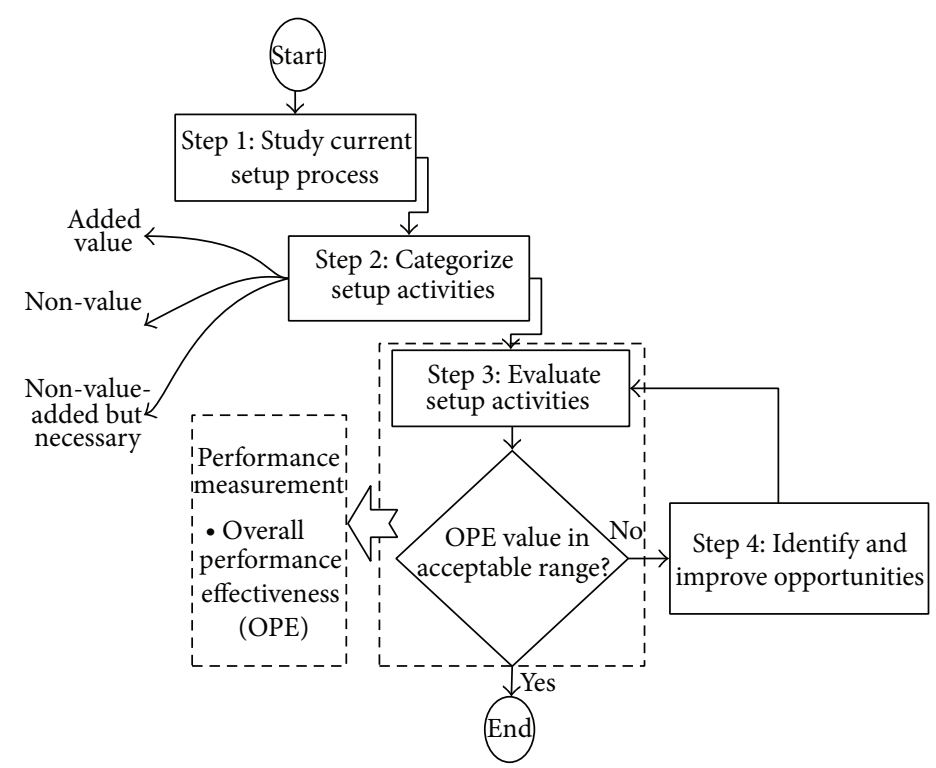

FIGURE 1: Setup improvement methodology.

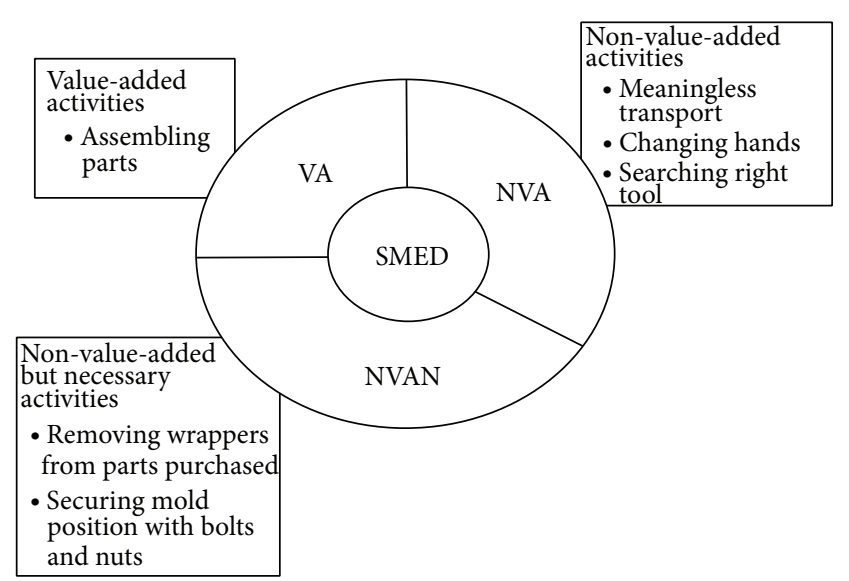

FIgURE 2: Categories of setup activities.

activities along with the reduction of setup time will result in a shorter effective cycle time per part. In this paper, the entire methodology was applied to a case study company, details of which are discussed in the following section.

\section{Case Study}

The case study reported in this paper took place in an electronic manufacturing plant. The plant has 50 IM machines operating 24 hours daily. Three eight-hour shifts are scheduled daily. Five setup technicians were responsible for the setup operation, and two technicians were assigned per team to complete the given setup task. Each team was able to set up an average of six machines every day. The number of molds in the storage was more than 300 sets, and the technicians were required to perform machine setup with various molds according to the production order given by the production

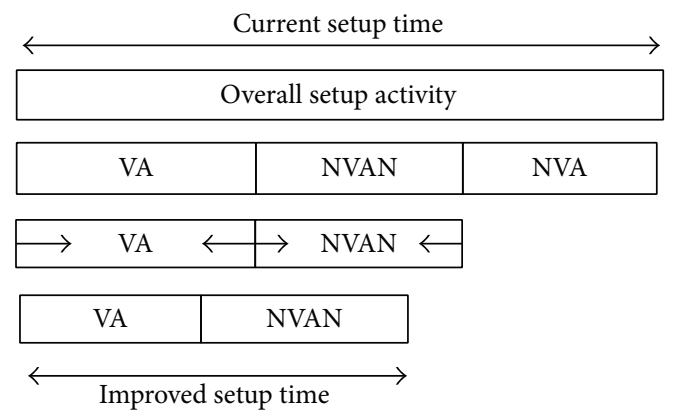

FIGURE 3: Setup time reduction.

management. The following sections discuss the applicability of the developed methodology based on the case study.

Step 1. (study current setup). In the study, machine 19 was chosen as the studied machine. Machine 19 has 20 tonnes of molding capacity. Compared with other available injection machines on the shop floor, machine 19 is considered as one of the smallest machines. It is also the most utilized machine, which allowed constant analysis of the setup procedures. Another main advantage of this machine setup is its relative similarity to most machines on the shop floor. Therefore, the study focused on obtaining setup information on machine 19 , such as overall setup procedures on the machine and time study on setup operation. To understand the current situation, the overall machine setup, from receiving the order for setup to the actual machine setup, was observed and recorded. Interviews were conducted with the personnel, such as the setup technicians, to further describe the plant's practices and standard operation procedures on current setup operations. A time study on the setup operation of machine 19 involving the setup technicians was also performed. The setup elements were observed. Table 1 lists the details of the time 
study on each recorded element. The entire setup operation required 43.21 minutes before the machine was ready for production.

Step 2. (categorize setup). Next, the setup operations involved were distinguished and divided into the following three components: VA, NVAN, and NVA activities. The last column of Table 1 exhibits all the proposed activities during a setup operation. From the categories, these activities were used to identify the causes of ineffectiveness of the machine setup. VA activities are defined as the activities that directly or indirectly contribute to the setup operation and allow the production to run as planned. In Table 1, lifting the new mold into the machine (Step 8) is considered as a VA activity in the setup operation. Installing the mold is a necessary action; otherwise, the mold will remain on the ground, where none of the procedures will show how the mold can be installed into the machine. On other hand, NVA activities are defined as activities that are unnecessary during the setup of the machine. For example, tool finding (Step 10) was supposed to be performed before the actual setup of machine 19. This action should not occur during setup because it will cause idling to the setup operation; even when no operations were done on the machine, time was still running. Meanwhile, NVAN activities ensure that the VA steps are properly completed. Table 1 reveals that fixing the mold clamps (Step 11) does not contribute to the product, but it is necessary to ensure clamps hold the mold at its position in the machine.

Step 3. (evaluate the setup operation). OPE is then used to determine the effectiveness of the current setup process. It is calculated according to the proposed mathematical method discussed in Step 3 in Section 2. An availability element and a performance element of 0.3224 and 0.4673 were obtained based on (1) and (3), respectively. The OPE value of the current setup process was $15.07 \%$, which was below the acceptable range. Therefore, further improvements on the current setup process were required.

Step 4. (identify improvement opportunities). An analysis of the steps involved in the setup revealed several reasons for the poor performance of setup operations. The highest contribute for the setup time is "find the new mold". First, the poor mold storage delayed the entire setup operation which is required 20 minutes. Technicians needed to check each tag on the molds to retrieve the required molds, which were poorly arranged in the storage room. No visual lines or benchmarks for mold placement also exist in the rack. The lack of proper standards in mold coding underlies the difficulties the technicians face as well as the longer time consumed for retrieving the required mold accurately and effectively. Second, the lack of an established checklist of equipment had lowered the standards for performing a setup operation. The technicians were not well equipped and often searched for missing tools during the setup operation, thus causing unwanted time wastage.

This study aims to improve the setup activities by incorporating a developed performance measurement. The developed performance measurement used to evaluate the effectiveness of performing a setup process is named overall performance effectiveness (OPE). OPE is a measurement index that shows the effectiveness of a setup process improvement. The paper is arranged as follows: Section 2 describes the methodology used to improve the setup process, Section 3 presents the case study and the results conducted in an injection molding (IM) company, Section 4 compares the results from Section 3, Section 5 discusses the results, and Section 6 concludes the paper.

The second highest contribute for the setup time is "adjust and align mold". The mold inlet needed to be adjusted in order to align it with the injection nozzle. For mold alignment activities, the technician consumes time to adjust the mold based on their vision and experience. Few test runs are needed for skilled technicians to attain accurate alignment until the best part is produced. The unskilled setup technician consumes even longer time as he performs a trial-anderror adjustment method because they lacked knowledge and training on adjusting molding machines.

Three alternatives were suggested to overcome the aforementioned problems:

(i) mold location line with mold number tag;

(ii) equipment list;

(iii) color cross-line indicator on mold and machine.

The first alternative was used to improve the moldhandling method in storage location. Molds were aligned with the colored tape on the mold storage rack and a mold number tag was used to identify the storage mold. Following the current mold master list, the molds were arranged according to the size, customer, and frequency of usage. Different colors may be used in the labels for different items to ensure that molds and equipment are kept in their designated places. This method will significantly reduce time lost finding the correct mold before setup. This alternative implies a simple visual management concept that can simplify and improve the means of delivery information. The technician in charge can easily determine the accurate location.

For the second alternative, a list of equipment checklist was initiated to ensure that no tools are lost before any setup operation. A checklist or check table can be a tool template with drawings. This list indicates the availability of all tools or parts required for a particular setup at a glance. It is used to confirm the availability of equipment before actual machine setup. For example, by checking equipment availability on a checklist before performing any machine setup, the technician instantly knows which equipment is unavailable in his equipment cart. Thus, the time to find unavailable tools during machine setup is reduced. This alternative also improves visibility to track the availability of a tool visually.

For the third alternative, a cross-line indicator was implemented to improve mold adjustment on the injection machine. A cross line was drawn on the mold fixture on the machine. The cross will intersect the nozzle center point. A corresponding cross line was drawn on the mold surface. Aligning both cross lines on the mold surface and the machine fixture reduces mold adjustment time. 
TABLE 1: Setup elements of machine 19.

\begin{tabular}{lccc}
\hline Step & Setup element & Element setup time (mins) & Category \\
NVAN & NVA \\
\hline 1 & Find new mold & 20.00 & $\sqrt{ }$ \\
2 & Get and shift mold from store to machine 19 & 3.00 & $\sqrt{ }$ \\
3 & Clean used mold (spray) & 0.50 & $\sqrt{ }$ \\
4 & Fix the crane clamp to use mold & 1.00 & $\sqrt{ }$ \\
5 & Remove four mold clamps & 1.50 & $\sqrt{ }$ \\
6 & Lift up used mold & 1.25 & $\sqrt{ }$ \\
7 & Move crane to new mold & 0.92 & $\sqrt{ }$ \\
8 & Lift up new mold & 1.67 & $\sqrt{ }$ \\
9 & Adjust and align mold & 7.50 & $\sqrt{ }$ \\
10 & Find and get tools & 1.00 & $\sqrt{ }$ \\
11 & Fix back mold clamps & 2.67 & $\sqrt{ }$ \\
\hline
\end{tabular}

TABLE 2: Data on the setup process before and after improvements.

\begin{tabular}{lcc}
\hline Data collected & Before improvement & After improvement \\
\hline Planned setup time (mins) & 20.00 & 20.00 \\
Actual total setup time (mins) & 43.24 & 21.00 \\
NVA time (mins) & 29.00 & 7.67 \\
\hline
\end{tabular}

\section{Comparison Result}

OPE was used to measure the performance effectiveness of a setup operation, and a performance comparison of the setup operation before and after the improvements was conducted. The OPE measurement comparison was employed to evaluate the setup operation before and after the improvements and to validate the improvements made. Improvements after the implementation of the suggested alternatives in Step 4 were tested in a pilot run. Table 2 shows the collected data of setup operations performance before and after improvement. Planned setup time is the total setup time suggested by the management to the setup team to perform a setup operation on a machine. Based on the complexity of the setup procedure for machine 19 and to remain coherent with the SMED concept of the company, the ideal setup time given by the management was 20 minutes. Actual total setup time was the actual total time spent on the machine setup based on the time study mentioned in Table 2 . The recorded setup time improved from 43.24 minutes to 21.00 minutes. The main contribution of the reduction of total setup time was an improvement in the NVA activities.

The total time spent on the NVA activities was reduced from 29.00 minutes to 7.67 minutes. Time spent on such activities was significantly reduced to $70 \%$. These NVA activities were the main focus for improvement in this study. As shown in Figure 4, improvements conducted in the three activities contributed significantly to improving the time spent on NVA activities. The time for finding the required mold improved from 20.00 minutes to 5.00 minutes, a $75 \%$ time reduction. Additionally, no tool finding activities occurred after the improvement, which eliminated the activities and reduced

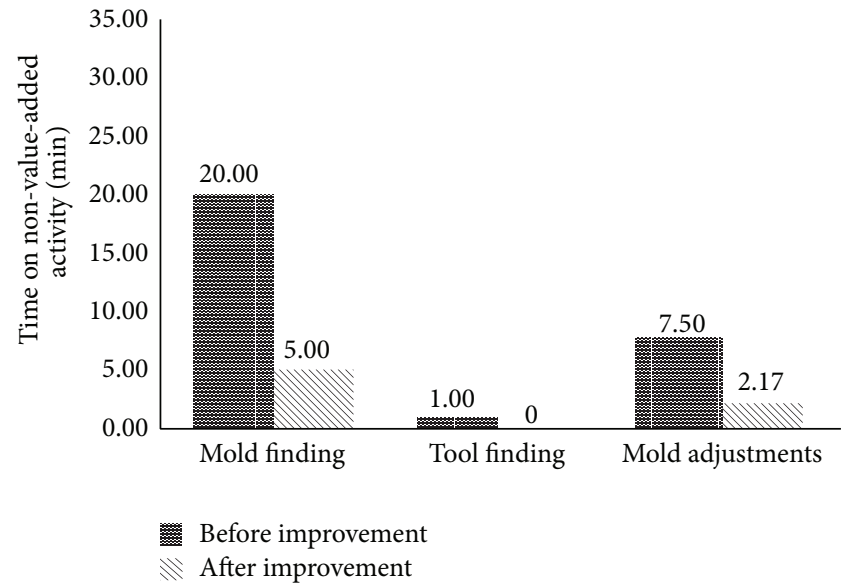

Figure 4: Comparison of list of NVA activities before and after improvement.

overall activity time by 1.00 minute. The mold adjustment step also had improved by $71 \%$ in its activity time, from 7.50 minutes to 2.17 minutes.

Figure 5 shows the OPE performance of machine 19 before and after improvement. Before the improvement, an OPE value of $15.07 \%$ indicated a poor performance by the technician in setup operations. After improvements were carried out on the setup operation of machine 19, OPE showed an increased performance efficiency of $60.45 \%$, which is inside the accepted percentage range mentioned in Step 3 of methodology section. The performance effectiveness of the setup process increased to more than $45 \%$ of its 


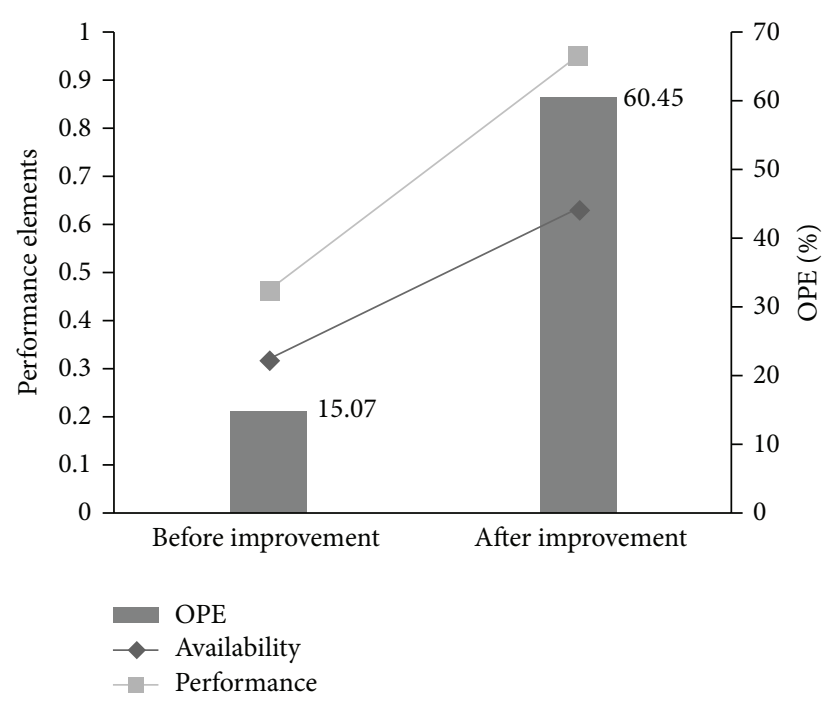

FIGURE 5: Results of OPE of machine 19.

original performance according to the proposed performance measurement. With this implementation of measurement, top management can better understand the performance of setup process on the machine, and indirectly evaluate and monitor the performance of the setup technician.

\section{Discussion}

OPE measurement makes it possible to facilitate the performance of a setup technician in setup procedures. For example, OPE can measure the efficiency of technicians in a setup operation. If the OPE level in a particular setup operation is lower than the recommended level, investigations can be done to determine the problem in the operation. Furthermore, OPE allows the management to optimize setup operations by facilitating potential improvement areas. It enables technicians to improve the operation procedures that have the most impact on the overall setup operation. Currently, SMED is focused on reducing the time spent on setup operations based on historical data. No real-time techniques can provide management swift feedback on the improvement made on a process. If historical records are unavailable, the improvement made will be untraceable. For such a situation, OPE is a useful application that will allow management to monitor and facilitate setup improvement without any reference to historical data. OPE also allows users to have better control over the entire setup operation. Performance on internal and external setups can be monitored individually.

OPE enables the management to have a better understanding on the performance of each element, and thus exercise better planning on SMED improvement for each element. Availability metrics were used to calculate the total time spent in NVA activities. It indicates the ratio of total time of VA and NVAN activities to the total setup time used on one machine. As the performance measurement is dependent on the total time spent on NVA activities, issues were raised regarding the necessity of taking NVAN activities into account. The definitions of the activities are regarded as important criteria to measure the reliability of the performance measurement. Performance of the operator in the setup operation was calculated as the discrepancy ratio of actual total setup time to ideal setup time.

In this case study, the use of a common mathematical approach to calculate performance efficiency as the ratio of actual setup time to planned setup time. It indicated that the result of performance measurement is inversely proportional to its performance. Therefore, the discrepancy value was used to lever the mathematical formula for performance measurement. This method can be used to calculate the performance of the setup technician in a setup operation on a machine and to meet the setup time requirement.

As stated by Van Goubergen and Van Landeghem [1], the reduction setup time impacts production throughput positively. The OPE can be as a guidance for offering a set up time improvement to shorten the production downtime for changeover purpose. Once the duration of the setup process shortened, the given time for running the production is increased; thus it will increase productivity.

\section{Conclusion}

The performance measurement indicator, OPE, provided useful results as a monitoring tool for setup performance. It provided useful information on the performance of an operator on setup operation, which was always undertaken in the SMED concept. With this indicator, management can monitor the setup performance and further enhance setup improvement from the operator's perspective. OPE provides useful guidelines to improve the inefficiencies in setup operations. This paper presented a case study and argued that, in the sequential application of stages of the setup reduction methodology, improvements should be measured to gauge the improved performance. In the case study, OPE was used and successfully showed the performance effectiveness of a setup technician after comparing the results before and after the setup improvements.

\section{Conflict of Interests}

The authors declare that there is no conflict of interests regarding the publication of this paper.

\section{Acknowledgments}

The authors acknowledge the Fundamental Research Grant Scheme (FRGS) and Knowledge Transfer Project (KTP) Research Grant provided by MOE for funding the study that resulted in this paper.

\section{References}

[1] D. van Goubergen and H. van Landeghem, "Rules for integrating fast changeover capabilities into new equipment design," Robotics and Computer-Integrated Manufacturing, vol. 18, no. 34, pp. 205-214, 2002. 
[2] L. Bamber and B. G. Dale, "Lean production: a study of application in a traditional manufacturing environment," Production Planning and Control, vol. 11, no. 3, pp. 291-298, 2000.

[3] S. Patel, P. Shaw, and B. G. Dale, "Setup time reduction and mistake proofing methods: a study of application in a small company," Business Process Management Journal, vol. 7, no. 1, pp. 65-75, 2001.

[4] M. Cakmakci and M. K. Karasu, "Set-up time reduction process and integrated predetermined time system MTM-UAS: a study of application in a large size company of automobile industry," International Journal of Advanced Manufacturing Technology, vol. 33, no. 3-4, pp. 334-344, 2007.

[5] A. C. Moreira and G. C. S. Pais, "Single minute exchange of die: a case study implementation," Journal of Technology Management and Innovation, vol. 6, no. 1, pp. 129-146, 2011.

[6] C. Moxham and R. Greatbanks, "Prerequisites for the implementation of the SMED methodology: a study in a textile processing environment," International Journal of Quality and Reliability Management, vol. 18, no. 4, pp. 404-414, 2001.

[7] B. Ulutas, "An application of SMED methodology," World Academy of Science, Engineering and Technology, vol. 55, pp. 100-103, 2011.

[8] M. Cakmakci, "Process improvement: performance analysis of the setup time reduction-SMED in the automobile industry," International Journal of Advanced Manufacturing Technology, vol. 41, no. 1-2, pp. 168-179, 2009.

[9] B. M. Deros, D. Mohamad, M. H. M. Idris, M. N. A. Rahman, J. A. Ghani, and A. R. Ismail, "Setup time reduction in an automotive battery assembly line," International Journal of Systems Applications, Engineering \& Development, vol. 5, no. 5, pp. 618-625, 2010.

[10] R. Domingos, B. Fernando, R. Sousa, and S. Carmo-Silva, "An application of the SMED methodology in an electric power controls company," in Proceedings of the International Conference on Innovations, Recent Trends And Challenges in Mechatronics, Mechanical Engineering and New High-Tech Products Development, vol. 3, pp. 47-55, 2011.

[11] T. Ohno, Toyota Production System: Beyond Large Scale Production, Productivity Press, 1988.

[12] M. Braglia, M. Frosolini, and F. Zammori, "Overall equipment effectiveness of a manufacturing line (OEEML): an integrated approach to assess systems performance," Journal of Manufacturing Technology Management, vol. 20, no. 1, pp. 8-29, 2009.

[13] K. Rahmani, M. P. Bonab, and M. K. Naghadeh, "Evaluating the overall effectiveness of production equipment and machinery," American Journal of Scientific Research, vol. 31, pp. 59-68, 2011. 

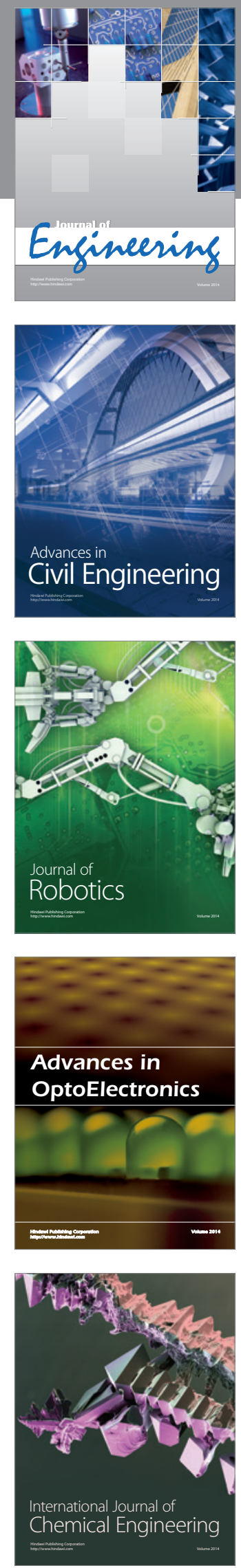

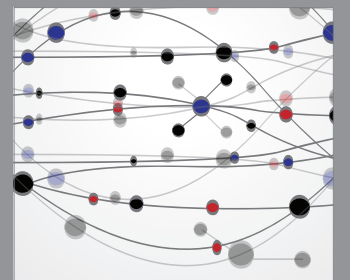

The Scientific World Journal
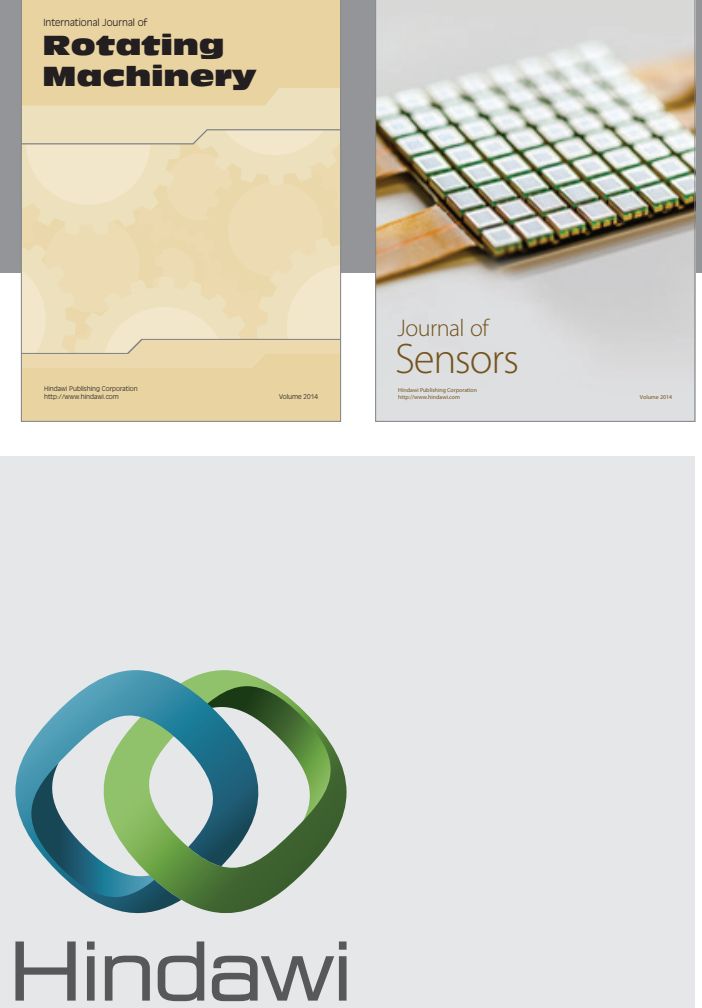

Submit your manuscripts at http://www.hindawi.com
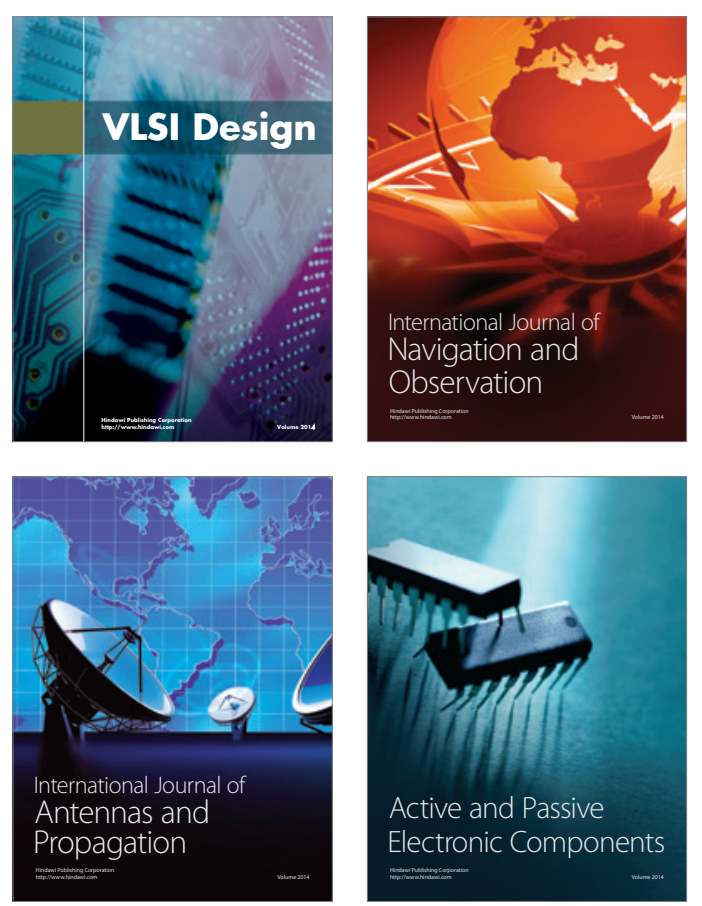
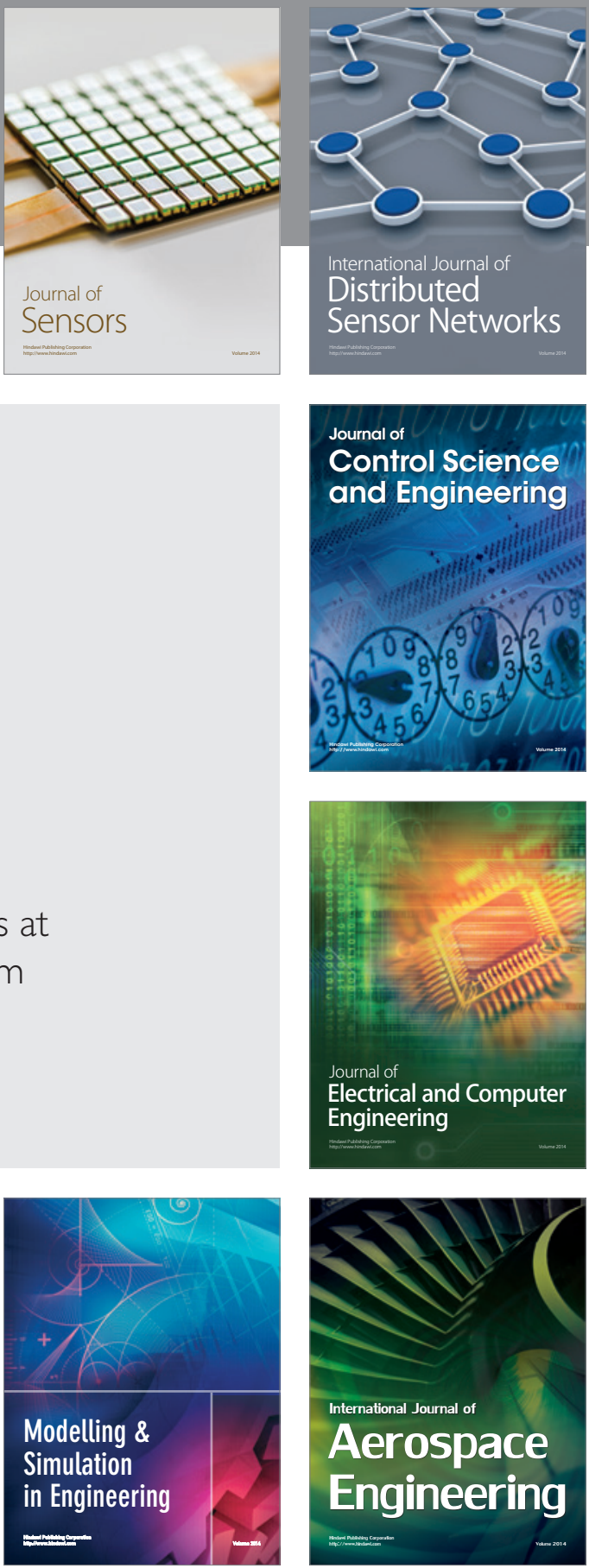

Journal of

Control Science

and Engineering
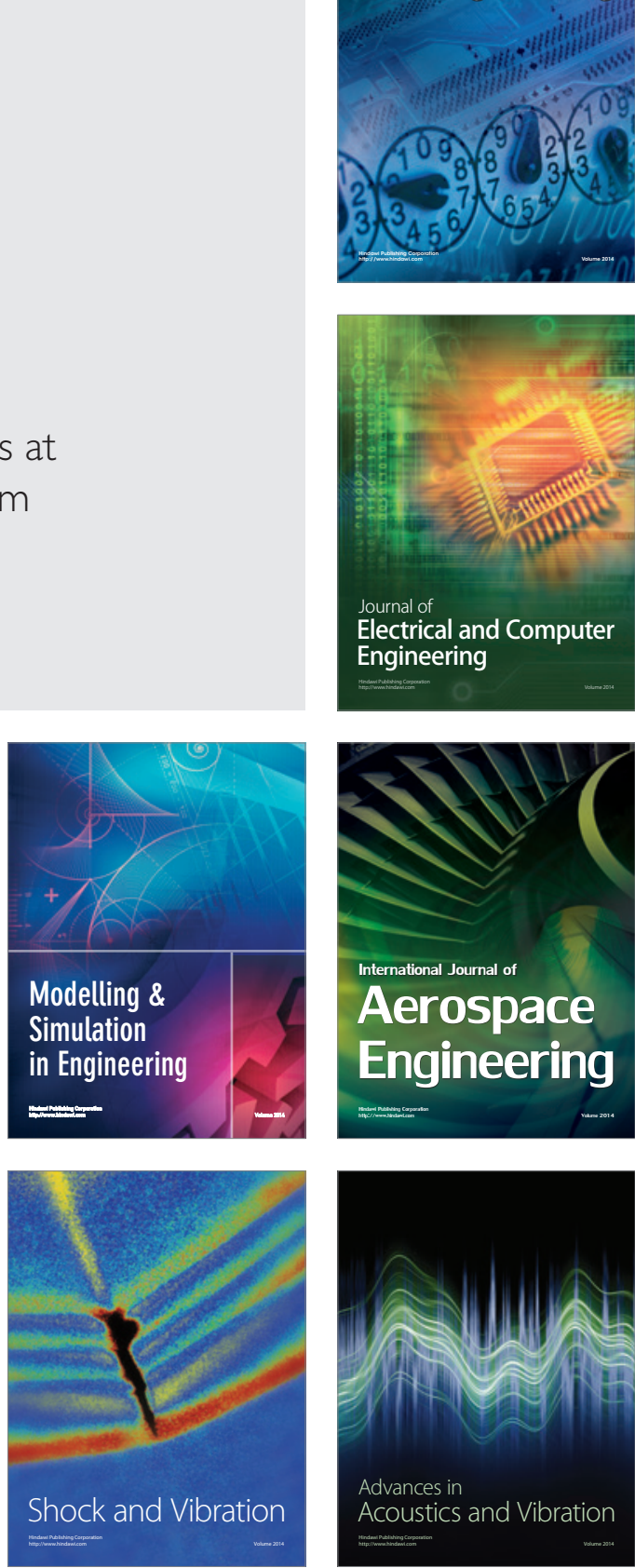